\section{Gender Differences in Mental Toughness and Coping with Injury in Runners}

\section{Pamela Andrews ${ }^{1}$ and Mark A Chen ${ }^{2 *}$}

\begin{abstract}
Objective: The aim of this study was to examine differences in Mental Toughness (MT) and coping with injury between male and female runners at different levels of experience.

Methods: 478 runners were categorized into beginners $(n=47)$ intermediate $(n=294)$ and advanced $(n=137)$. All participants had sustained an injury during their running career. The present study utilized Survey Monkey ${ }^{\mathrm{TM}}$, which is an online survey collection service. Participants completed two questionnaires the Psychological performance inventory (PPI-A) which measured MT and the Coping Inventory for Competitive Sport (CICS), which measured task, distancing and disengagement coping strategies.

Results: MANCOVA revealed that males had significantly higher total MT $(p<0.001)$ and composite MT than females $(p=0.001)$ Further ANCOVA analysis revealed that males had significantly higher self belief $(p=0.001)$ and visualization $(p=0.002)$ compared to females. With regards to coping, males had statistically higher composite task orientated coping than females $(p<0.001)$ and further ANCOVA analysis showed that males used more mental imagery $(p<0.001)$ to deal with injury than females. There was no difference between genders in terms of distancing orientated coping However, MANCOVA did reveal a difference in the disengagement coping composite $(p<0.001)$ and further ANCOVA analysis found that females used more disengagement and resignation coping $(p=0.00002)$ compared to males.
\end{abstract}

Conclusion: This study has provided evidence for how MT differences between male and female runners may be related to different coping strategies to deal with injury. More specifically males used more task orientated coping whereas females used more disengagement coping in order to deal with injury. Further research should examine how injury appraisal is related to different coping strategies in males and females.

\section{Keywords}

Mental toughness; Injury; Coping; Multivariate analysis

\section{Introduction}

A widely reported concept described within the literature is that of Mental Toughness (MT). MT is a multidimensional construct often highlighted as a positive and essential component to successful sporting performance [1]. Recent studies [2-5] have examined how

\footnotetext{
*Corresponding author: Mark A Chen, Department of Sport and Exercise Science, School of Social Sciences, Business and Law, University of Teesside, Borough Road, Middlesbrough, TS1 3BA, United Kingdom, Tel: 01642342326 Fax: 01642342399; E-mail: m.chen@tees.ac.uk
}

Received: October 24, 2014 Accepted: December 08, 2014 Published: December 14, 2014
MT and coping with performance related stress may differentiate performers. However, relatively little is known about how MT relates to dealing with the stress of injury. According to current research [6] MT can be defined in terms of four components: Determination is the athletes resolve, sense of commitment and dedication to playing and practicing their sport. Self Belief, describes the athletes confidence and use of positive affirmations. Positive Cognition includes self regulatory feelings such as thought control, energy and enjoyment, and visualization is the athlete's use of positive visualization skills in training and competition. This definition of MT provides a basis to explore differences between genders in how they cope with the injury experience.

Gender differences in MT and coping have been found in previous studies with regard to performance [2]. One study found that males scored significantly higher than females on total MT (Cohen's $\mathrm{d}=0.33$ ), challenge, control emotions, control life and confidence ability [2]. Research using the SMTQ, found that male athletes scored significantly higher than female athletes on total MT, confidence and control [7]. In exploring the relationship between MT and coping they found low to moderate correlations in $8 / 10$ subscales of the CICS. It could be reasoned that these coping processes utilized by males may lead to differences in coping with injury compared to females.

Recent studies have found that athletes from a range of team and individual sports perceive sports injury to be a stressor $[3,8]$ that requires additional coping resources. Coping has been defined as "a constantly changing cognitive and behavioral effort to manage specific external and/or internal demands that are appraised as taxing or exceeding the resources of the person" [9]. At the macro level coping strategies have been separated into two higher order dimensions. Task orientated coping refers to actions that are employed in order to change or master some aspects of an event that is perceived as stressful. This dimension includes specific coping strategies to manage a stressor such as planning and logical analysis. The second dimension, avoidance orientated coping, includes behavior to disengage oneself from the task or social diversion onto task-irrelevant cues. It has been suggested that this coping orientation may lead to an individual suppressing some warnings of possible injury or reporting fewer injuries [10]. These findings suggest that higher levels of MT may lead to athletes under reporting and under estimating their injuries. As males are consistently shown to have higher MT than females it could be reasoned that they employ different coping strategies. No research to date has investigated the specific coping strategies that may underlie any differences between genders in response to injury.

Some studies examining gender differences in coping have implications for dealing with injury. Research has found that female cross-country runners used less task orientated coping strategies than did their male counterparts [11]. More recent work examining gender differences in the appraisal and coping process also used endurance athletes [8]. Data was collected the day before and on a competitive race day for all participants. Results indicated females perceived less control and more venting of emotions on the day of the race and males reported higher usage of suppression of competing activities and lower use of instrumental social support compared 
to female runners. As females may have less perceived control than males, this may lead to avoidance coping being an adaptive short term strategy during injury, as this type of coping may be preferred when the individual has limited control [12]. However, no research to date has examined how the stress of time off training and competition due to injury relates to potentially different coping responses of males and females.

Therefore, the aim of this study was to examine differences in MT and coping with injury between male and female runners.

\section{Methodology}

\section{Participants}

478 runners within the United Kingdom and Ireland agreed to participate in this retrospective study (Males $n=284$, Females $n=194)$ aged between 18 and $78(\mathrm{M}=42.0(\mathrm{SD}=11.6))$ with running experience ranging from 2 months to 30 years. The sample consisted of runners from beginner $(n=47)$, intermediate $(n=294)$ and advanced $(n=137)$ levels. All participants had sustained an injury during their running career. The University research ethics committee approved this research and all participants gave informed consent prior to participation.

\section{Measures}

Demographic information: The demographic data sheet consisted of questions concerning the participant's gender, age, how long they have been running, level of runner and how long they were unable to train or compete in running due to injury. An informed consent form was issued to explain in further detail the purpose of the study.

Psychological Performance Inventory-A: The PPI-A [6] was utilized to measure MT. The PPI-A is a 14-item measure, with an overall score of MT with 4 subscales of determination, self-belief, positive cognition and visualization. Each item is scored on a 5-point likert scale ranging from almost always to almost never. Confirmatory factor analysis (CFA) demonstrated good support for the PPI-A [6]. The PPI-A was found to have acceptable internal consistency $(\alpha>0.70)$ $[6,13]$. Recent support has been found for the confirmatory validity of the PPI-A with regard to achievement goals [4].

The Coping Inventory for Competitive Sport: The (CICS) [14], was used to evaluate coping levels in injured runners. The CICS is a 39 -item measure with 10 subscales. The 10 coping strategy subscales are further grouped into three second order dimensions of [1] task orientated coping, (mental imagery, logical analysis, relaxation, thought control, energy expenditure, social support) [2] distraction orientated coping (distancing and mental distraction) and [3] disengagement-orientated coping (venting of unpleasant emotions and disengagement/resignation). As the original items were intended for competition, some of the items were reworded to represent running and injuries sustained. Each item is scored on a 5-point likert scale ranging from "does not correspond at all" to "corresponds very strongly". Adequate reliability has been demonstrated for the CICS, Cronbach's index of internal consistency ranging between 0.67 and 0.87 [14]. CFA has demonstrated good support for the structure of the CICS [14]. Support has also been found for the construct validity of the three-second order dimensions of coping [15].

\section{Procedure}

Participants were recruited by contacting running clubs and associations throughout the United Kingdom and Ireland. A letter was emailed to club officials requesting their involvement in the study. The present study utilized the software program Survey Monkey ${ }^{\mathrm{m}}$, which is an online survey collection service. Recent research has suggested on-line surveys are considered to produce a better response rate within most sport psychology research [16]. Once the inventories were imputed to the program an electronic link was generated, which was then emailed to participants. The data was then collected within the Survey Monkey ${ }^{m}$ database for further analysis. The letter sent out to club officials included the link to the inventories, which if they agreed to participate, was forwarded onto their members. If the members consented to participate they could click on the link where they completed a series of 3 questionnaires, which were completed at their own leisure. All participants were requested to be completely honest in their responses, individuals were also assured that their participation within the study was voluntary and their data was confidential. Participants were also aware that if they wanted to withdraw from the study at any time they could do so without any consequence. The responses from each participant were then coded for further analysis.

\section{Data analysis}

Data was first screened for outliers and normality of all dependent variables. All variables were within an acceptable range of normality. It was found that the males were significantly older and had more months running training than females. Therefore, these, along with time off injured were entered as covariates in subsequent analysis. We therefore conducted ANCOVA and MANCOVA with gender as the fixed factor and MT and coping as the dependent variables with age, years running and time out injured as the covariates in all analysis. Four 1-way ANCOVA tests were conducted on the total MT, task orientated coping, distraction and disengagement orientated coping scores. Four 1-way MANCOVA tests were done on the subscales of each of the inventories total scores. Follow up ANOVA was conducted in the instance of significant main effects using a bonferroni correction for type I errors $(p<0.008)$. Following recent guidelines, both partial eta effect sizes and cohen's d effect sizes were reported [17]. Each main effect is reported with the partial eta effect size and 95\% confidence intervals calculated [18]. Also, 95\% confidence intervals on each mean gender difference within levels of experience were calculated using a customized spreadsheet [19]. Cohen's d effect sizes were calculated using the formula, $d=$ mean difference / $\sqrt{ }$ MSE.

\section{Results}

Independent $t$ tests showed that males were older $\mathrm{t}(454.8)=5.606$, $\mathrm{p}<0.001$ than females and had significantly more years running $t$ $(439.4)=2.058, p=0.044)$ but did not show a significant difference in total months off injured between gender $\mathrm{t}(476)=0.879, \mathrm{p}=0.380$. All three variables were entered as covariates in all subsequent analysis (Table 1).

The descriptive statistics for total MT and the four MT subscales are presented in Table 2. Using Wilks $\lambda$, a ANCOVA with covariates age $\mathrm{F}(1,473)=1.535, \mathrm{p}=0.216$, partial $\eta^{2}=0.00395 \%$ CI $[0.000,0.021]$

\begin{tabular}{|l|l|l|l|}
\hline & Age & Years Running & Total Time Injured \\
\hline Males & $44.3(11.9)$ & $14.3(12.0)$ & $4.2(10.0)$ \\
\hline Females & $38.5(10.1)$ & $12.1(11.0)$ & $3.5(5.2)$ \\
\hline
\end{tabular}

Table 1: Mean and standard deviation of age, years running and total time off injured (months) for males and females. 
and years running $\mathrm{F}(1,473)=3.288, \mathrm{p}=0.070$, partial $\eta^{2}=0.007$ $[0.000,0.0283]$ and total time off $\mathrm{F}(1,473)=1.136, \mathrm{p}=0.287$, partial $\eta^{2}=0.002[0.000,0.0189]$ and MT total as the dependent variable revealed a significant main effect of gender $F(1,473)=15.492$, $\mathrm{p}<0.001$, partial $\eta^{2}=0.032[0.008,0.070]$. This showed that males had a statistically higher level of total MT than females.

A MANCOVA analysis with age $\mathrm{F}(4,470)=5.290, \mathrm{p}<0.001$, partial $\eta^{2}=0.043[0.000,0.037]$, years running $\mathrm{F}(4,470)=1.810, \mathrm{p}=0.126$, partial $\eta^{2}=0.015[0.000,0.022]$ and total time off $\mathrm{aF}(4,470)=0.462$, $\mathrm{p}=0.764$, partial $\eta^{2}=0.004[0.000,0.0126]$ as covariates and the four MT subscales as the dependent variables revealed a statistical main effect of Gender $\mathrm{F}(4,470)=4.564, \mathrm{p}=0.001$, partial $\eta^{2}=0.037,95 \%$ CI: $[0.006,0.069]$. Follow up Individual ANOVA's on the subscales, showed that males were statistically higher than females in self belief, $F(1,473)=11.368, p=0.001$, partial $\eta^{2}=0.023[0.003,0.057]$ and visualization $\mathrm{F}(1,473)=9.187, \mathrm{p}=0.003$, partial $\eta^{2}=0.019[0.002,0.050]$.

The descriptive statistics for the 6 subscales of task orientated coping are presented in Table 3 . Using Wilks $\lambda$, ANCOVA with age $\mathrm{F}(1,473)=4.258, \mathrm{p}=0.040$, partial $\eta^{2}=0.009[0.000,0.033]$ and months running, $\mathrm{F}(1,473)=0.101, \mathrm{p}=0.751$, partial $\eta^{2}=0.000[0.000,0.010]$ and timeout as $\mathrm{F}(1,473)=0.083, \mathrm{p}=0.083$,partial $\eta^{2}=0.000[0.000,0.075]$ the covariates with task orientated coping total as the DV revealed no statistical difference between gender $\mathrm{F}(1,473)=2.827, \mathrm{p}=0.096$, partial $\eta^{2}=0.006[0.000,0.027]$. A MANCOVA analysis with the coping subscales as the dependent variables with age $\mathrm{F}(6,468)=5.769$, $\mathrm{p}=0.000$, partial $\eta^{2}=0.069[0.023,0.106]$, years running as the covariates $\mathrm{F}(6,468)=0.708, \mathrm{p}=0.643$, partial $\eta^{2}=0.009[0.000,0.017]$ and total time off $\mathrm{F}(6,468)=0.669, \mathrm{p}=0.674$, partial $\eta^{2}=0.009[0.000,0.0175]$ revealed a significant main effect of Gender $F(6,468)=4.126$, $p<0.001$, partial $\eta^{2}=0.050[0.010,0.082]$, with males scoring statistically higher than females. Mental imagery $F(1,473)=12.297, p<0.001$, partial $\eta^{2}=0.025$ $[0.005,0.059]$ showed that males were statistically higher than females.

The descriptive statistics for the 4 subscales of distancing and disengagement orientated coping are presented in Table 4. Using Wilks $\lambda$, ANCOVA with age $\mathrm{F}(1,473)=8.794, \mathrm{p}=0.003$, partial $\left.\eta^{2}=0.018[0.002,0.049]\right)$, years running $\mathrm{F}(1,473)=0.051, \mathrm{p}=0.821$, partial $\left.\eta^{2}=0.000[0.000,0.008]\right)$ and total time off $\mathrm{F}(1,473)=1.799$, $\mathrm{p}=0.180$, partial $\eta^{2}=0.004[0.000,0.0224]$ as the covariates and distancing orientated coping total as the dependent variable revealed no statistical difference between gender $\mathrm{F}(1,473)=0.860$, $\mathrm{p}=0.354$, partial $\eta^{2}=0.02[0.000,0.018]$. The MANCOVA with age $\mathrm{F}(2,472)=8.070, p=0.001$, partial $\eta^{2}=0.033 \quad[0.007,0.069]$, years running $\mathrm{F}(2,472)=0.083, \mathrm{p}=0.920$, partial $\eta^{2}=0.000[0.000,0.004]$ and total time off $\mathrm{F}(2,472)=1.478, \mathrm{p}=0.229$, partial $\eta^{2}=0.006[0.000,0.0253]$ as the covariates and the two distancing orientated coping subscales as the dependent variables revealed no statistical main effect of Gender $\mathrm{F}(2,472)=0.264, \mathrm{p}=0.768$, partial $\eta^{2}=0.001[0.000,0.011]$ (Table 4).

Using Wilks $\lambda$, a ANCOVA with age $F(1,473)=26.747, \mathrm{p}<0.001$, partial $\eta^{2}=0.054[0.003,0.050]$, years running $\mathrm{F}(1,473)=1.232, \mathrm{p}=0.268$, partial $\left.\eta^{2}=0.003[0.000,0.009]\right)$ and total time off $\mathrm{F}(1,473)=0.466$,

\begin{tabular}{|c|c|c|c|c|c|c|}
\hline \multirow[t]{2}{*}{ TM } & \multicolumn{2}{|c|}{ Beginners } & \multicolumn{2}{|c|}{ Intermediate } & \multicolumn{2}{|c|}{ Advanced } \\
\hline & Mean Diff $95 \% \mathrm{Cl}$ & Effect size & Mean Diff $95 \% \mathrm{Cl}$ & Effect size & Mean Diff $95 \% \mathrm{Cl}$ & Effect size \\
\hline MT total & $1.1+/-4.8$ & 0.16 & $1.8+/-1.7$ & 0.26 & $2.2+/-2.2$ & 0.31 \\
\hline Determination & $-0.7+/-1.3$ & -0.38 & $0.2+/-0.4$ & 0.11 & $-0.2+/-0.6$ & -0.11 \\
\hline Self belief & $0.3+/-2.0$ & 0.11 & $1.0+/-0.6$ & 0.37 & $1.1+/-2.0$ & 0.41 \\
\hline Positive cog & $0.9+/-1.1$ & 0.38 & $0.2+/-0.6$ & 0.08 & $0.5+/-1.1$ & 0.21 \\
\hline Visualization & $0.6+/-1.8$ & 0.19 & $0.5+/-0.7$ & 0.16 & $0.8+/-1.2$ & 0.26 \\
\hline
\end{tabular}

Table 2: Mean difference with $95 \%$ confidence intervals and effect size statistics. Gender is the independent factor split into levels of experience with MT total and 4 subscales are the dependent variables.

\begin{tabular}{|c|c|c|c|c|c|c|}
\hline & \multicolumn{2}{|c|}{ Beginners } & \multicolumn{2}{|c|}{ Intermediate } & \multicolumn{2}{|c|}{ Advanced } \\
\hline & Mean Diff $95 \% \mathrm{Cl}$ & Effect size & Mean Diff $95 \% \mathrm{Cl}$ & Effect size & Mean Diff 95\% Cl & Effect size \\
\hline Task coping & $2.3+/-7.5$ & 0.17 & $1.1+/-3.2$ & 0.08 & $0.1+/-5.2$ & 0.01 \\
\hline Mental imagery & $1.4+/-1.5$ & 0.13 & $0.8+/-0.7$ & 0.25 & $-0.3+/-1.2$ & -0.09 \\
\hline Logical analysis & $0.3+/-1.8$ & 0.10 & $0.4+/-0.7$ & 0.13 & $0.5+/-1.1$ & 0.16 \\
\hline Relaxation & $-0.9+/-2.2$ & -0.24 & $0.2+/-0.7$ & 0.05 & $0.3+/-1.4$ & 0.08 \\
\hline Thought control & $1.9+/-1.7$ & 0.60 & $0.4+/-0.7$ & 0.13 & $0.1+/-1.2$ & 0.04 \\
\hline Energy expenditure & $0.2+/-1.4$ & 0.08 & $0.3+/-0.6$ & 0.12 & $0.0+/-0.9$ & 0.04 \\
\hline Social support & $-1.6+/-2.1$ & -0.46 & $-1.0+/-0.8$ & -0.29 & $-0.9+/-1.3$ & -0.26 \\
\hline
\end{tabular}

Table 3: Mean difference with $95 \%$ confidence intervals and effect size statistics. Gender is the independent factor split into levels of experience with task orientated coping total and 6 task coping subscales are the dependent variables.

\begin{tabular}{|c|c|c|c|c|c|c|}
\hline & \multicolumn{2}{|c|}{ Beginners } & \multicolumn{2}{|c|}{ Intermediate } & \multicolumn{2}{|c|}{ Advanced } \\
\hline & Mean diff $95 \% \mathrm{CI}$ & Effect size & Mean diff $95 \% \mathrm{Cl}$ & Effect size & Mean diff $95 \% \mathrm{Cl}$ & Effect size \\
\hline Distancing coping & $1.1+/-2.9$ & 0.22 & $-1.2+/-1.2$ & -0.24 & $-2.0+/-1.7$ & 0.41 \\
\hline Distancing & $0.8+/-1.4$ & 0.35 & $-0.2+/-0.5$ & -0.09 & $-0.8+/-0.9$ & -0.35 \\
\hline Mental distraction & $0.3+/-1.8$ & 0.10 & $0.4+/-0.7$ & 0.13 & $0.5+/-1.1$ & 0.16 \\
\hline $\begin{array}{l}\text { Disengagement } \\
\text { coping }\end{array}$ & $-0.7+/-4.2$ & -0.12 & $-1.7+/-1.4$ & -0.28 & $-0.6+/-2.3$ & -0.10 \\
\hline Venting of emotions & $0.1+/-2.4$ & 0.03 & $0.1+/-0.9$ & 0.03 & $1.5+/-1.6$ & 0.39 \\
\hline $\begin{array}{l}\text { Disengagement \& } \\
\text { resignation }\end{array}$ & $-0.3+/-2.1$ & -0.10 & $-1.7+/-0.7$ & -0.55 & $-1.1+/-1.1$ & -0.36 \\
\hline
\end{tabular}

Table 4: Mean difference with $95 \%$ confidence intervals and effect size statistics. Gender is split according to level of experience with distancing orientated coping total, disengagement orientated coping total and 4 distancing/disengagement coping subscales as dependent variables. 
$\mathrm{p}=0.495$, partial $\eta^{2}=0.001[0.000,0.0015]$ as the covariates and disengagement orientated coping total as the dependent variable revealed no statistical difference between gender $\mathrm{F}(1,473)=0.337$, $\mathrm{p}=0.562$, partial $\eta^{2}=0.001[0.000,0.018]$. The MANCOVA with age $\mathrm{F}(2,472)=17.977, \mathrm{p}<0.001$, partial $\eta^{2}=0.071[0.010,0.096]$ and years running $\mathrm{F}(2,472)=0.822, \mathrm{p}=0.440$, partial $\eta^{2}=0.003[0.000,0.018]$ and total time off $\mathrm{F}(2,472)=0.246, \mathrm{p}=0.782$, partial $\eta^{2}=0.000[0.001,0.005]$ as the covariates and the two disengagement orientated coping subscales as the dependent variables revealed a statistical main effect of Gender $\mathrm{F}(2,472)=23.786, \mathrm{p}<0.001$, partial $\eta^{2}=0.092[0.046,0.141]$. However only the disengagement and resignation $\mathrm{F}(1,474)=18.391$, $\mathrm{p}=0.00002$, partial $\eta^{2}=0.037[0.010,0.076]$ showed that females were statistically higher than males.

\section{Discussion}

The aim of this study was to examine gender differences in MT and coping with injury. There were three important findings. Differences were found between gender in total, composite MT and the self belief and visualization subscales. Also, males showed higher task orientated coping than females, whereas females showed higher disengagement coping relative to males.

First, the study found that with regard to total (Cohen's $d=0.23$ ) and composite MT, that males scored statistically higher than females after controlling for age, months running and total time off injured, thus replicating previous findings as measured by the MTQ48 $[2,3,5]$. Between levels of experience, there was a slight increase in male to female differences from beginner (Cohen's $\mathrm{d}=0.16$ ) to advanced levels (Cohen's $\mathrm{d}=0.31$ ) of participation as reflected in the mean differences and effect sizes. The data indicates that differences between genders in MT total were largest for the advanced participation level. The amount of variance accounted for by age and years running was small, similar to previous work which controlled for similar variables [5]. The results indicate that learning experience and/or biological changes cannot account for gender differences in total MT. Both self belief and visualization were both greater for males compared to females therefore, it appears there are specific aspects of MT which differ between the genders. Previous work found males scored higher in challenge, control emotions, control life and confidence ability [5]. Challenge and confidence overlap with self belief while visualization appears to be tapping into something of importance when related to coping with injury as mental imagery was also different between genders as measured by the CICS. Although, It should be borne in mind these results pertain to sport performance rather than dealing with injury, previous findings exploring differences in confidence between genders did find that males were higher [20] therefore this study adds support to this line of reasoning.

Males scored higher than females on task composite coping, although only mental imagery reached significance. These task coping composite results, are similar to those previously found [8] that females felt less in control before a competitive race compared to males (Cohen's $\mathrm{d}=0.03$ ). Those authors were able to ascertain that the primary appraisal of the stressful event was similar between genders [8], whereas the present study cannot ascertain how the time off due to injury was appraised in the present study. Therefore, the interpretation that the coping difference is due to gender rather than differences in the way the time out due to injury was appraised should be treated with caution. However, the mental imagery difference is consistent with the visualization MT subscale being higher for males. The mental imagery items in the CICS had items such as 'visualized myself getting better' and 'rehearsed the execution of my injury 'whereas the visualization items on the PPI-A concerned visualizing tough situations prior to competition and ability to think in pictures about their sport. These results would seem to suggest that being a good visualizer for sport performance might transfer over to visualizing recovery from sports injury. Mental imagery and visualization can be conceived as active, problem solving approaches that enhance optimism regarding future outcomes. One of the highest correlations in previous studies [2] was the correlation between mental imagery and optimism $(r=0.29)$. Therefore, male's greater usage of mental imagery to cope with injury may have consequences for differences in perceptions of recovery. It may also have been that the mentally tougher males subsequently perceived their injury to be less stressful and therefore were more optimistic [21] relative to females.

The task coping effect sizes indicate that the social support subscale is worth mentioning. Although it did not reach significance, the social support effect sizes from beginner (Cohen's $d=0.46$ ) to advanced (Cohen's $\mathrm{d}=0.26$ ) were larger than most other subscales. Also, the finding that females tend to use more social support than males is supported in the literature [22,23]. Social support was the only task oriented coping subscale where females were greater than males. The results infer that females tended to ask others for advice concerning their injury more than males. Despite the task composite result showing greater males scores, the social support data indicates that certain discrete social support behaviors within task coping, may be more likely exhibited by females rather than males and that caution should be displayed in interpreting the task composite result. It could well have been that different task coping behaviors exhibited by males and females cancelled each other out [23].

There was no significant difference between genders in total or composite distancing coping. These items measured the degree to which individuals attempt to distance themselves from other runners and use distraction coping. The two distancing coping subscales of distancing and mental distraction can be interpreted as a form of avoidance coping. The data suggests that males, although mentally tougher may not ignore the injury more than females as has been suggested [8].

However, there were statistical differences between genders in composite disengagement coping, which showed females to have higher levels than males. In particular the disengagement and resignation subscale showed that females were higher than males. The items on this subscale assess the degree to which the individual had lost hope of attaining their goals, hopelessness, discouragement and wishing the injury would go away. These results are similar to previous studies $[6,22]$ who found that females reported more venting of emotions on race day and used more wishful thinking to cope with stress. Likewise, the disengagement scale had items concerning 'wishing the injury would go away' and feeling hopeless and discouraged. It could be expected higher amounts of hopelessness may be expected in the current sample if the injury was preventing training or competing. Also, it has been found that resignation is negatively correlated with MT while pessimism positively correlated with MT [3] and as females had less total MT and self belief than males, they would likely react with more resignation when unable to train or compete due to injury. However, these results should be treated with caution due to the low effect sizes.

There are some weaknesses with the study. First, the design was 
retrospective with regard to the assessment of the injury stressor. Second, this study did not consider the appraisal of the injury and the specific nature of the stress induced by the injury, therefore it is unknown how this affected the coping responses. Future studies should use a prospective design to examine how appraisal is related to the coping responses. Third, as participants filled out the questionnaires, at different times in their running and competitive schedule this may have influenced both the MT and coping responses.

In summary, this study aimed to examine gender differences in MT and coping with injury using a retrospective method with competitive runners. The coping results indicated that males tended more toward dealing with their injuries using more task focused coping, specifically mental imagery, which is perhaps supported by their increased usage of visualization for sport performance compared to females. Despite this broad task coping difference, females did use more social support than males, especially for beginners, perhaps suggesting that females employed a narrower range of problem solving strategies than males when dealing with being sidelined. In contrast, females used more disengagement strategies and tended toward giving up and wishing the injury would go away, which may be related to their relatively lower total MT scores. This supports the literature claim that females use more emotion focused coping. Therefore, this study has found that differences between genders in MT with regard to performance may also apply to coping with injury. However as the relative success of the coping was not ascertained, further research should seek to examine short and long term psychological adaptations to injury and subsequent performance using prospective designs.

\section{References}

1. Golby J and Sheard M (2004) Mental toughness and hardiness at differen levels of rugby league. Pers Individ Dif 37: 933-942.

2. Nicholls AR, Polman RCJ, Levy, AR, Backhouse SH (2008) Mental toughness, optimism, pessimism, and coping among athletes. Pers Individ Dif 44: 1182-1192.

3. Kaiseler M,Polman R,Nicholls A (2009) Mental toughness, stress, stress appraisal, coping and coping effectiveness in sport. Pers Individ Dif 47: 728-733.

4. Gucciardi DF(2012) Measuring mental toughness in sport: a psychometric examination of the psychological performance inventory-a and its predecessor. J Pers Assess 94: 393-403.

5. Nicholls AR,Polman RCJ,Levy AR,Backhouse SH (2009) Mental toughness in sport: Achievement level, gender, age, experience, and sport type differences. Pers Individ Dif 47: 73-75.

6. Golby J,Sheard M,Van Wersch A (2007) Evaluating the factor structure of the psychological performance inventory (PPI). Percept Mot Skills 105: 309-325.

7. Sheard M,Golby J,Van Wersch, A (2009) Progress toward construct validation of the sport mental toughness questionnaire (SMTQ). Eur J Psychol Assess 25: 186-193.

8. Hammermeister J,Burton D (2004) Gender differences in coping with endurance sport stress: Are men from mars and women from venus? J Sport Behav 27: 148-165.

9. Lazarus RS, Folkman S (1984) Stress, appraisal and coping. Springer Publishing Company, New York.

10. Carson F, Polman RC (2009) The facilitative nature of avoidance coping within sports injury rehabilitation. Scand J Med Sci Sports 20: 235-240.

11. Madden CC,Kirkby RJ,McDonald D (1989) Coping styles of competitive middle distance runners. Int J Sport Psychol 20: 287-296.

12. Carver CS,Scheier MF,Weintraub JK (1989) Assessing coping strategies: A theoretically based approach. J Pers Soc Psychol 56: 267-283.

13. Sheard M (2010) Mental toughness: The mindset behind sporting achievement. Routledge.
14. Gaudreau P, Blondin JP (2002) Development of a questionnaire for the assessment of coping strategies employed by athletes in competitive sports settings. Psychology of Sport and Exercise 3: 1-34.

15. Hancock GR (2001) Effect size, power, and sample size determination for structured means modeling and MIMIC approaches to between-groups hypothesis testing of means on a single latent construct. Psychometrika 66: 373-388.

16. Lonsdale C, Hodge K, Rose EA (2006) Pixels vs. Paper: Comparing Online and Traditional Survey Methods in Sport Psychology. J Sport Exerc Psychol 28: 100-108.

17. Fritz CO, Morris PE, Richler JJ (2012) Effect size estimates: Current use calculations and interpretation. J Exp Psychol Gen 141: 2-18.

18. Smithson M (2003) Confidence intervals. Sage, Thousand Oaks, CA.

19. Cummings $G$ (2012) Introduction to the new statistics: Effect sizes, meta analysis and confidence intervals. Routledge, New York.

20. Krane V, Williams JM (1994) Cognitive anxiety, somatic anxiety and confidence in track and field athletes: the impact of gender, competitive level and task characteristics. Int J Sport Psychol 25: 203-217.

21. Wadey R, Evans L, Hanton S, Neil R (2012) An examination of hardiness throughout the sport injury process. Br J Health Psychol 17: 103-128.

22. Kaiseler M, Polman RCJ, Nicholls AR (2012) Gender differences in appraisal and coping: An examination of the situational and dispositional hypothesis. Int J Sport Psychol 43: 1-14

23. Tamras LK, Janicki D, Helgeson V (2002) Sex differences in coping behavior: A meta-analytic review and an examination of relative coping. Pers Soc Psychol Rev 6: 2-30.

\section{Author Affiliation}

${ }^{1}$ Musculoskeletal and Neurological Rehabilitation, School of Health and Life Sciences, Glasgow Caledonian University, Cowcaddens Road, Glasgow, G4 $O B A, U K$

${ }^{2}$ Department of Sport and Exercise Science, School of Social Science Business and Law, University of Teesside, Middlesbrough, TS1 3BA, UK

Submit your next manuscript and get advantages of SciTechnol submissions

* 50 Journals

* 21 Day rapid review process

* 1000 Editorial team

* 2 Million readers

* Publication immediately after acceptance

* Quality and quick editorial, review processing

Submit your next manuscript at • www.scitechnol.com/submission 